\title{
INTENSIDADE E ÉPOCAS DE PODA VERDE EM PEREIRA 'ABATE FETEL' SOBRE DOIS PORTA-ENXERTOS
}

\author{
LEO RUFATO ${ }^{2}$, JOSÉ LUIZ MARCON FILHO ${ }^{3}$, GILMAR ARDUINO BETTIO MARODIN ${ }^{4}$, \\ AIKE ANNELIESE KRETZSCHMAR ${ }^{5}$, DAVID JOSÉ MIQUELUTI ${ }^{6}$
}

RESUMO - O Brasil é um dos maiores importadores mundiais de pera europeias. A falta de material genético adaptado e a deficiência de tecnologias de manejo, bem como o abortamento de gemas florais das cultivares europeias plantadas têm desestimulado os produtores, ocasionando a redução de áreas plantadas. O objetivo deste estudo foi estabelecer uma metodologia de poda verde na cultivar Abate Fetel a fim de aumentar a frutificação e controlar o vigor das plantas. O trabalho foi desenvolvido nos pomares da Frutirol Agrícola Ltda., em Vacaria-RS, nas safras de 2006/2007 e 2007/2008. Utilizou-se a cultivar Abate Fetel sobre dois porta enxertos: marmeleiros 'Adams' e 'EMC'. Foram avaliados os efeitos de dois níveis de poda verde em três diferentes épocas de realização. Os tratamentos consistiram na poda verde, com retirada de 1/3 e 2/3 do comprimento dos ramos do ano, nas épocas de janeiro, fevereiro e março, e uma testemunha, sem poda verde. O delineamento adotado foi o de blocos ao acaso, com parcelas subsubdivididas, com cinco repetições e duas plantas por repetição. Conclui-se que o porta-enxerto 'EMC' induz melhor controle de vigor e aumenta os índices de produtividade para a cv Abate Fetel, comparado com o marmeleiro 'Adams'. A poda verde, com intensidade de $2 / 3$ de retirada dos ramos, independentemente do mês de realização, aumenta a produtividade da pereira cv Abate Fetel.

Termos de Indexação: Pyrus communis L., Cydonia oblonga Mill., Marmelo 'Adams', Marmelo 'EMC', Abbè Fetel.

\section{INTENSITY AND PERIODS OF SUMMER PRUNING IN 'ABATE FETEL' PEAR TREE ON TWO ROOTSTOCKS}

\begin{abstract}
Brazil is one of the world's largest importers of European pear. The lack of adapted genetic material and deficiency of management technologies, as well the abortion of flower buds of European cultivars has discouraged producers, causing a decrease on planted areas. The purpose of this study was to establish a summer pruning methodology for the cultivar Abate Fetel aiming the increase of fruit set and control of plant vigor. The study was conducted in Frutirol Agricola Ltd. orchards, placed in Vacaria / RS, harvest of 2006/2007 and 2007/2008. The cultivar Abate Fetel was used on two rootstocks: quince 'Adams' and 'EMC'. Were evaluated the effects of two summer pruning levels on three different periods. Treatments consisted of summer pruning with removal of $1 / 3$ and $2 / 3$ of shoots length, on January, February, March and a control without pruning. The experimental design adopted was randomized blocks with split plots, five replications and two plants per replication. It was concluded that the rootstock 'EMC' induces better vigor control and increases productivity rates for 'Abate Fetel', than quince 'Adams'. The summer pruning with removal of $2 / 3$ of shoots length, regardless the month of realization, increases the productivity of Abate Fetel pear.
\end{abstract} Index terms: Pyrus communis L., Cydonia oblonga Mill., Quince ‘Adams', Quince 'EMC', Abbè Fetel.

\footnotetext{
${ }^{1}$ (Trabalho 257-11). Recebido em: 07-011-2011. Aceito para publicação em: 10-04-2012.

${ }^{2}$ Dr. Fruticultura, Professor CAV/UDESC; Avenida Luiz de Camões, 2090, Bairro Conta Dinheiro, Lages-SC, CEP: 88520-000. Email: leoruffato@yahoo.com.br

${ }^{3}$ Mestrando em Produção Vegetal CAV-UDESC; Bolsista Capes. E-mail: marconfilho_jl@yahoo.com.br

${ }^{4}$ Eng. Agr. Dr., Professor Associado II do Dep. Horticultura e Silvicultura, Faculdade de Agronomia, UFRGS, Porto Alegre-RS. Email: marodin@ufrgs.br; bolsista CNPq.

${ }^{5}$ Orientadora, Dra. Fruticultura, Professora CAV/UDESC. E-mail: a2aak@cav.udesc.br

${ }^{6}$ Eng. Agr. Dr., Professor CAV/UDESC. E-mail: a2djm@cav.udesc.br
} 


\section{INTRODUÇÃO}

Entre as frutas de clima temperado, a pera é a terceira mais consumida e importada pelo Brasil (RUFATO et al., 2011). Apesar do grande mercado interno para a pera, a cultura não apresenta destaque entre as frutíferas de clima temperado cultivadas no Brasil, sendo a espécie a de menor expressão em termos de área cultivada, produção e valor da produção (PEREIRA; HERTER, 2010).

O Brasil é o segundo maior importador mundial de peras. Em 2008, foram importadas 139.777,53 toneladas com gastos na importação de US\$ $120.624,142$ mil. Esses valores significam 54,8\% da quantidade total de frutas importadas e $49,6 \%$ do valor pago, respectivamente (IBRAF, 2010).

Originária do sul da França, a pera 'Abate Fetel' é a segunda mais consumida no continente europeu, e suas plantas são caracterizadas por apresentarem médio vigor, com boa afinidade de enxertia com marmeleiros e produtividade elevada (PREDIERI et al., 2005). Entre as principais características do fruto estão a forma alongada, coloração amarelo-esverdeada e polpa de textura fina, doce e suculenta. Além da valorização no mercado internacional, outra vantagem para os fruticultores é que a 'Abate Fetel' pode ser armazenada por longos períodos, podendo ser mantida por até quatro meses em frigoconservação (PREDIERI; GATTI, 2009; CALVO et al., 2011).

A expansão da cultura da pereira no Brasil é limitada por vários fatores, dentre os quais, destaca-se como principal a falta de material genético e a deficiência de tecnologias de manejo, o que tem dificultado a produção de peras nas condições do Sul do Brasil e limitando a expansão do cultivo (TREVISAN et al., 2005).

Outro fator limitante no desenvolvimento da cultura da pereira, segundo Giacobbo et al. (2007), relaciona-se à insuficiência de estudos sobre porta-enxertos. Atualmente, as poucas áreas implantadas com pereira, no Brasil, estão em sua maioria sobre o porta-enxerto Pyrus calleryana, o qual proporciona às plantas alto vigor de crescimento, que, como consequência, dificulta a condução das plantas e seu manejo cultural.

Os marmeleiros vêm sendo utilizados como porta-enxertos preferenciais para a pereira, com o intuito de proporcionar plantas de pequeno porte e rápida frutificação, além de proporcionarem uniformidade aos pomares (PIO et al., 2008; STERN et al., 2009; MILOSEVIC; MILOSEVIC, 2011). No entanto, existem poucas informações no Brasil sobre o uso de marmeleiros como porta-enxertos ananizantes, assim como sua relação no controle do vigor das plantas e a respectiva melhoria na eficiência produtiva da pereira.

Dentre os marmeleiros utilizados como porta-enxerto para a pereira, destacam-se o 'Marmeleiro C' ('EMC') e o marmeleiro 'Adams'. O 'EMC', selecionado e propagado livre de vírus pela Estação Experimental "East-Malling" (Inglaterra), é o clone mais ananizante, induzindo elevada produtividade $\mathrm{e}$ precocidade. O marmeleiro 'Adams', por sua vez, é originário da Bélgica, sendo um intermediário entre 'Marmeleiro C'e 'Marmeleiro A', o qual é caracterizado por apresentar baixo vigor, indução da produção precoce, elevada produtividade e eficiência produtiva (JACKSON, 2003).

O crescimento da pereira e de outras frutíferas é influenciado por fenômenos de competição por fotoassimilados entre a parte vegetativa, órgãos de frutificação e o restante da planta. Os ramos, brotos e folhas são drenos mais fortes de fotoassimilados, o que prejudica toda a parte reprodutiva, como a formação de gemas florais e frutos. $\mathrm{O}$ excesso de crescimento vegetativo e uma escassa diferenciação floral podem determinar uma planta pouco produtiva.

A quase totalidade das podas das plantas de folhas caducas é realizada no inverno, entretanto há a necessidade de intervir frequentemente com poda verde durante o período vegetativo. A finalidade principal das intervenções nesta época do ano é regular o desenvolvimento dos novos ramos na planta durante o decorrer do ciclo (SOARES et al., 2003; ASÍN et al., 2007), pois a poda drástica de inverno favorece um forte crescimento vegetativo e reduz o número de gemas floríferas.

Assim, o objetivo deste trabalho foi estudar o comportamento vegetativo:produtivo da pereira cv. Abate Fetel submetida a duas intensidades de poda verde no verão, enxertada sobre dois marmeleiros.

\section{MATERIAL E MÉTODOS}

O presente trabalho foi desenvolvido nos pomares da empresa Frutirol Agrícola Ltda., Município de Vacaria-RS, localizado na região dos Campos de Cima da Serra, estando o pomar situado à latitude sul $29^{\circ} 32^{\prime}$, longitude oeste $50^{\circ} 54^{\prime}$ e altitude média de 955 metros.

O experimento foi instalado em dezembro de 2006, num pomar de 'Abate Fetel', sobre dois porta-enxertos de marmeleiros: 'Adams' e 'EMC', com sete anos de idade. As plantas foram conduzidas em líder central, com espaçamento de 0,8 metro entre plantas e 3,0 metros entre filas, e os tratos culturais (superação de dormência e tratamentos fitossanitários) foram realizados segundo as recomendações 
usuais da cultura.

Foram estudados os efeitos de dois níveis de poda verde, em três diferentes épocas de realização de poda. O delineamento adotado foi o de blocos ao acaso, com parcelas subsubdivididas, com cinco repetições e duas plantas por repetição. O fator porta-enxerto compôs as parcelas, o fator poda verde foi estudado nas subparcelas, constituindo-se de redução de $1 / 3$ e $2 / 3$ do comprimento de ramos do ano, com diâmetro não inferior a seis milímetros e testemunha, sem poda verde. As épocas, janeiro, fevereiro e março constituíram as subsubparcelas.

A poda verde foi executada na primeira semana dos meses correspondentes ao tratamento de época, nos anos de 2007 e 2008.

Nas safras de 2006/2007 e 2007/2008, no período de dormência, foram avaliadas as seguintes variáveis: a) incremento do tronco $\left(\mathrm{cm}^{2}\right)$, determinada através da subtração dos valores encontrados da área da seção do tronco, obtida através da média das medidas longitudinal e transversal à linha de plantio, a $20 \mathrm{~cm}$ do solo, calculada através da fórmula $\mathrm{A}=$ $\left(\pi \mathrm{d}^{2}\right) / 4$, em que, $\mathrm{d}=$ diâmetro; b) volume da copa $\left(\mathrm{m}^{3}\right)$, obtido pelas medidas da largura, espessura e altura da copa a partir do ponto de inserção do primeiro ramo no tronco; c) comprimento dos ramos do ano $(\mathrm{cm})$, obtido pela média da medida longitudinal de cinco ramos por planta; d) índice de fertilidade, através da relação entre o número de gemas floríferas e o comprimento do ramo do ano; e) estrutura formada no local da poda, (vegetativa ou florífera), sendo o resultado expresso em percentagem de estruturas de frutificação (Figura 2); f) Relação Volume copa/Área da seção do tronco $\left(\mathrm{m}^{3} \mathrm{~cm}^{-2}\right)$.

No período vegetativo, em fevereiro de 2008, foram avaliadas as variáveis: a) número de frutos por planta, obtido pela contagem dos frutos uma semana antes da colheita; b) produtividade estimada (ton ha${ }^{1}$ ), calculada pela multiplicação do peso total colhido por planta pelo número de plantas existentes em um hectare, de acordo com o espaçamento utilizado (3,0 x $0,8 \mathrm{~m}=4.166$ plantas $\mathrm{ha}^{-1}$ ); c) eficiência produtiva $\left(\mathrm{kg} \mathrm{m}^{-3}\right)$, calculada através da relação entre a produção por planta e o volume da copa; d) Relação Área tronco $/ \mathrm{N}^{\circ}$ Frutos $\left(\mathrm{cm}^{2}\right.$ fruto $\left.{ }^{-1}\right)$.

Devido à ocorrência de geada tardia no mês de outubro de 2008, que ocasionou abortamento de $100 \%$ dos frutos, não foi possível avaliar os atributos produtivos: produtividade estimada, eficiência produtiva e relação da área da seção do tronco por número de frutos para o ano de 2009.

As análises estatísticas foram conduzidas a partir dos dados de todas as unidades experimentais incluídas nos tratamentos estudados, de acordo com o delineamento experimental utilizado. Foi adotado um modelo linear de análise de variância, e as estimativas dos parâmetros foram testadas pelo teste F. As comparações de médias foram efetuadas por meio do teste de Tukey. As variáveis expressas em percentagem foram transformadas, elevando-se os seus valores unitários à potência $1 / 2$ e obtendo-se, a seguir, a função seno inversa (transformação arco-seno), conforme sugerido pela análise descritiva dos dados. Porém, os resultados são apresentados na escala original desta variável.

Todas as análises foram procedidas usando-se o programa estatístico WinStat. Para todos os testes efetuados, foi considerado o nível mínimo de significância de 5\%.

\section{RESULTADOS E DISCUSSÃO}

O comprimento dos ramos do ano das pereiras 'Abate Fetel' enxertadas sobre marmeleiro 'Ada$\mathrm{ms}$ ' foi superior àquelas enxertadas sobre marmeleiro 'EMC', na safra de 2006/2007. No entanto, na safra de 2007/2008, não houve diferença significativa entre os dois porta-enxertos (Tabela 1).

$\mathrm{O}$ incremento do tronco também foi superior nas plantas enxertadas sobre o marmeleiro 'Adams' e não houve diferença significativa para esta variável nas diferentes intensidades de poda, nos dois anos de estudo (Tabela 1). Os marmeleiros são classificados como porta-enxertos anões para pereiras, não somente pelo fato de pertencerem a outro gênero, o que pode acarretar menor desenvolvimento vegetativo devido à maior incompatibilidade, mas também pelo fato de absorverem menor quantidade de nitrogênio do que os porta-enxertos de Pyrus betulaefolia e Pyrus calleryana (BAKSHI; SINGH, 2010). No entanto, comparando os dois marmeleiros, o porta-enxerto 'Adams' apresenta maior vigor do que o porta-enxerto 'EMC', concordando com os resultados encontrados por Pio et al. (2008), os quais observaram que o comprimento médio da brotação de enxertos, aos 150 dias, foi maior no marmeleiro 'Adams' em relação ao 'EMC'.

Para as variáveis volume de copa e volume de copa/área da seção do tronco, não houve diferença significativa entre porta-enxertos (Tabela 1). Em relação a essas mesmas variáveis, a intensidade de poda de $2 / 3$ dos ramos apresentou menor valor (Tabela 1). Conforme Hartmann et al. (1990), um volume da copa reduzido acarreta melhor balanço vegetativo/ reprodutivo da planta, aumentando a frutificação .

As plantas enxertadas sobre o marmeleiro 'EMC' tiveram maior índice de fertilidade na safra de 2006/2007, em relação ao 'Adams', sendo 
não significativo em 2007/2008 (Tabela 2). Tal comportamento deve-se, provavelmente, ao menor crescimento vegetativo das plantas, melhorando o balanço entre a parte vegetativa e a reprodutiva (HARTMANN et al., 1990).

As podas verdes aumentaram significativamente a formação de estruturas de frutificação nas plantas enxertadas sobre o marmeleiro 'EMC'. Não houve diferença para esta variável em relação à época de realização da poda. No entanto, para o fator intensidade, plantas submetidas à poda de $1 / 3$ dos ramos do ano, aumentaram o número de estruturas de frutificação nos ramos remanescentes (Figura 1). Segundo Jackson (2003), a poda incrementa a disponibilidade de nitrogênio por gema e estimula o crescimento próximo ao ponto de corte, além de que, quando se poda parte de um ramo, estimula-se intensamente a região próxima do corte. Adicionalmente, de acordo com Soares et al. (2003), a eliminação do meristema terminal, através da poda, irá fomentar a diferenciação floral de gemas próximas e promover a formação de gemas florais de formação pronta ou antecipada, ou seja, no próprio ramo do ano.

Comparando os atributos produtivos da pereira cv Abate Fetel na safra de 2007/2008, número de frutos, produtividade estimada e eficiência produtiva (Tabela 3 ), as plantas enxertadas sobre o marmeleiro 'EMC' apresentaram resultados superiores àquelas enxertadas sobre marmeleiro 'Adams'. $\mathrm{O}$ resultado mais expressivo a destacar foi a produtividade estimada, onde o 'EMC' foi cerca de seis vezes superior ao 'Adams'.

O porta-enxerto utilizado em pereira afeta profundamente o comportamento da cultivar e pode proporcionar diferença de até $50 \%$ ou mais no rendimento de uma mesma cultivar; além disso, o porta-enxerto pode afetar o rendimento por unidade de tamanho da planta (produtividade). As causas destes efeitos sobre o rendimento não são sempre aparentes, mas podem estar, muitas vezes, relacionadas com diferenças em tolerância a diferentes solos, resistência a pragas e doenças ou absorção de nutrientes (BAKSHI; SINGH, 2010). A correta definição dos porta-enxertos e as respectivas cultivares-copa é fundamental para o sucesso de um pomar, pois os equívocos são de difícil correção e muito onerosos.

Com relação ao efeito da intensidade de poda sobre os parâmetros produtivos, a retirada de $2 / 3$ dos ramos aumentou o número de frutos por planta e, consequentemente, a produtividade estimada. A intensidade de poda de $2 / 3$ proporcionou rendimentos de $4 \mathrm{t} \mathrm{ha}^{-1}$ a mais do que a de $1 / 3$, e essa não diferiu significativamente das plantas-testemunha (Tabela 4).

De forma geral, o crescimento vegetativo e a diferenciação floral devem estar em equilíbrio para que as plantas sejam produtivas. $\mathrm{O}$ uso de porta-enxertos adequado e um eficiente manejo de poda são essenciais para atingir esse objetivo.

Mesmo que os resultados sejam ainda preliminares, pois os reflexos de intervenções de poda devem ser avaliados ao longo dos anos, parece que a utilização do porta-enxerto 'EMC' e a poda de $2 / 3$ do comprimento dos ramos do ano induzem pereiras 'Abate Fetel' a um adequado equilíbrio entre a parte vegetativa e a produtiva, com expectativas positivas de produtividade dessa pera europeia, nas condições do Sul do Brasil.
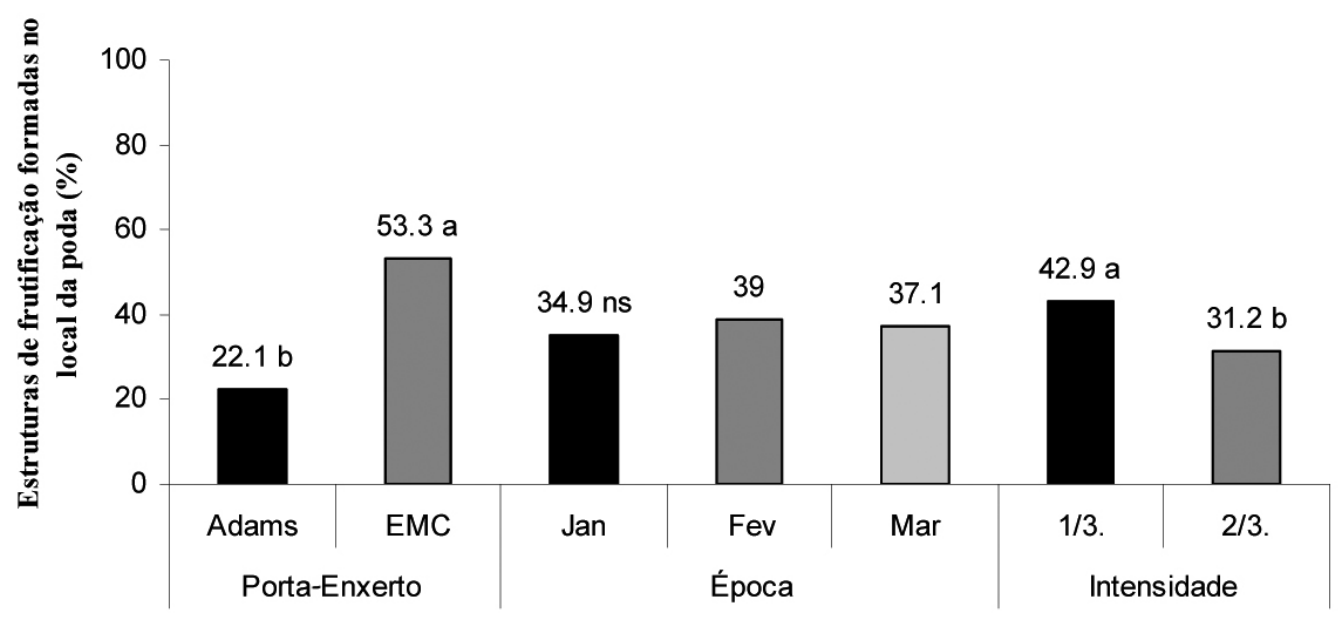

FIGURA 1 - Efeito da poda verde em pereiras 'Abate Fetel' enxertadas sobre marmeleiros 'Adams' e 'EMC': Estrutura de frutificação formada no local da poda. Safra 2007/2008. Vacaria/2011. 


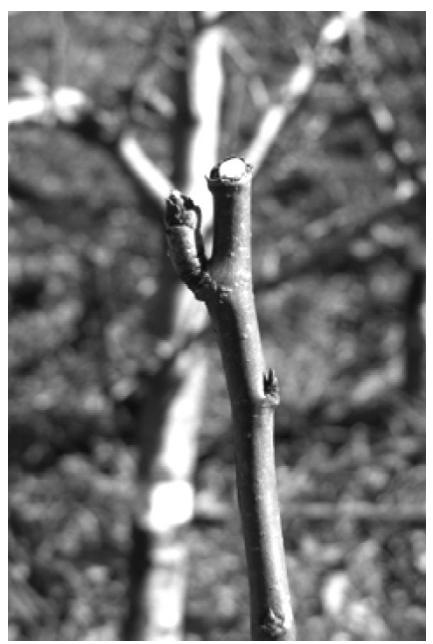

FIGURA 2 - Estrutura de frutificação formada no local da poda em pereira 'Abate Fetel' enxertada sobre o marmeleiro 'EMC' Safra 2007/2008. Vacaria/2011.

TABELA 1 - Efeito da poda verde em pereiras 'Abate Fetel' enxertadas sobre marmeleiros 'Adams' e 'EMC': Parâmetros de análise de vigor (comprimento dos ramos do ano; incremento do tronco; volume da copa; relação volume da copa e área do tronco). Vacaria/2011.

\begin{tabular}{|c|c|c|c|c|c|c|c|}
\hline \multicolumn{8}{|c|}{ 2006-2007 } \\
\hline \multirow{2}{*}{ Variáveis } & \multicolumn{3}{|c|}{ Porta-Enxerto } & \multicolumn{4}{|c|}{ Intensidade } \\
\hline & Adams & EMC & CV (\%) & $1 / 3$ & $2 / 3$ & Sem poda & CV (\%) \\
\hline Comprimento Ramo (cm) & $39,53 \mathrm{a}$ & $24,76 b$ & 31,73 & $31,97 \mathrm{~b}$ & $26,39 \mathrm{~b}$ & $38,08 \mathrm{a}$ & 20,46 \\
\hline Incremento do tronco $\left(\mathrm{cm}^{2}\right)$ & $0,9 \mathrm{a}$ & $0,00 \mathrm{~b}$ & 30,3 & $0,00 \mathrm{~ns}$ & 0,27 & 0,66 & 32,84 \\
\hline \multicolumn{8}{|c|}{$2007-2008$} \\
\hline \multirow{2}{*}{ Variáveis } & \multicolumn{3}{|c|}{ Porta-Enxerto } & \multicolumn{4}{|c|}{ Intensidade } \\
\hline & Adams & EMC & CV (\%) & $1 / 3$ & $2 / 3$ & Sem poda & CV $(\%)$ \\
\hline Comprimento Ramo (cm) & $26,27 \mathrm{~ns}$ & 25,99 & 12,39 & $23,61 \mathrm{c}$ & $25,89 \mathrm{~b}$ & $28,9 \mathrm{a}$ & 10,34 \\
\hline Incremento do tronco $\left(\mathrm{cm}^{2}\right)$ & $13,2 \mathrm{a}$ & $9,45 \mathrm{~b}$ & 26,7 & $11,59 \mathrm{~ns}$ & 11,33 & 11,05 & 20,29 \\
\hline Volume copa $\left(\mathbf{m}^{3}\right)$ & $2,6 \mathrm{~ns}$ & 3,5 & 15,04 & $3,16 a b$ & $2,88 \mathrm{~b}$ & $3,25 \mathrm{a}$ & 15,58 \\
\hline Volume copa/Seção tronco $\left(\mathrm{m}^{3} . \mathrm{cm}\right.$ & $0,073 \mathrm{~ns}$ & 0,096 & 14,51 & $0,087 \mathrm{a}$ & $0,078 \mathrm{~b}$ & 0,089 a & 14,24 \\
\hline
\end{tabular}

* Médias seguidas pela mesma letra na linha não diferem entre sí, pelo teste de Tukey, em nível de $5 \%$ de probabilidade de erro.

** ns = não significativo pelo teste de Tukey, em nível de 5\% de probabilidade de erro.

TABELA 2 - Efeito da poda verde em pereiras 'Abate Fetel' enxertadas sobre marmeleiros 'Adams' e 'EMC': Índice de fertilidade. Vacaria/2011.

\begin{tabular}{|c|c|c|c|c|}
\hline \multirow{2}{*}{ Porta-Enxerto } & \multicolumn{4}{|c|}{$2006 / 2007$} \\
\hline & $1 / 3$ & $2 / 3$ & Sem poda & Média \\
\hline Adams & $0,34 \mathrm{~b} \mathrm{~B}$ & $0,38 \mathrm{~b} \mathrm{AB}$ & 0,42 ns A & $0,38 \mathrm{~b}$ \\
\hline EMC & 0,47 a NS & $0,48 \mathrm{a}$ & 0,46 & $0,47 \mathrm{a}$ \\
\hline \multirow[t]{3}{*}{ CV (\%) } & & 15,76 & & 18,03 \\
\hline & \multicolumn{4}{|c|}{$2007 / 2008$} \\
\hline & $1 / 3$ & $2 / 3$ & Sem poda & Média \\
\hline Adams & $0,41 \mathrm{~ns} \mathrm{AB}$ & $0,40 \mathrm{~ns} B$ & 0,43 a $\mathrm{A}$ & $0,42 \mathrm{~ns}$ \\
\hline EMC & $0,42 \mathrm{~A}$ & $0,42 \mathrm{~A}$ & $0,38 \mathrm{~b} \mathrm{~B}$ & 0,41 \\
\hline CV (\%) & & 5,95 & & 5,09 \\
\hline
\end{tabular}

*Médias com letra minúscula iguais na coluna

e maiúscula iguais na linha não diferem entre si, pelo teste de Tukey, em nível de $5 \%$ de probabilidade de erro.

*** ns (NS) = não significativo pelo teste de Tukey, em nível de 5\% de probabilidade de erro. 
TABELA 3 - Atributos produtivos de pereiras 'Abate Fetel' enxertadas sobre marmeleiros 'Adams' e 'EMC': Número de frutos; Relação entre a área da seção do tronco e número de frutos; produtividade estimada e eficiência produtiva. Safra 2007/2008. Vacaria/2011.

\begin{tabular}{|c|c|c|c|}
\hline \multirow{2}{*}{ Variáveis } & \multicolumn{3}{|c|}{ Porta-Enxerto } \\
\hline & Adams & EMC & CV $(\%)$ \\
\hline Número de frutos & $4,10 \mathrm{~b}$ & $24,50 \mathrm{a}$ & 19,64 \\
\hline Seção tronco/ $\mathbf{N}^{0}$ Frutos $\left(\mathrm{cm}^{2}\right.$.fruto $\left.{ }^{-1}\right)$ & $7,52 \mathrm{a}$ & $1,21 \mathrm{~b}$ & 24,32 \\
\hline Produtividade Estimada (ton.ha-1) & $4,24 \mathrm{~b}$ & $26,9 \mathrm{a}$ & 43,9 \\
\hline Eficiência produtiva $\left(\mathrm{kg} \cdot \mathrm{m}^{-3}\right)$ & $0,39 \mathrm{~b}$ & $1,62 \mathrm{a}$ & 37,66 \\
\hline
\end{tabular}

*Médias seguidas pela mesma letra na linha não diferem entre si, pelo teste de Tukey, em nível de 5\% de probabilidade de erro.

TABELA 4 - Efeito da poda verde em pereiras 'Abate Fetel' enxertadas sobre marmeleiros 'Adams' e 'EMC': Atributos produtivos (número de frutos; relação entre a área da seção do tronco e número de frutos; produtividade estimada e eficiência produtiva). Safra 2007/2008. Vacaria/2011.

\begin{tabular}{|c|c|c|c|c|c|c|c|c|}
\hline \multirow{2}{*}{ Variáveis } & \multicolumn{4}{|c|}{ Época } & \multicolumn{4}{|c|}{ Intensidade } \\
\hline & Jan. & Fev. & Mar. & CV (\%) & $1 / 3$ & $2 / 3$ & Sem poda & CV (\%) \\
\hline Número de frutos & $11,3 \mathrm{~b}$ & $13,4 \mathrm{a}$ & $11,7 \mathrm{~b}$ & 8,33 & $10,7 \mathrm{~b}$ & $15,5 \mathrm{a}$ & $10,2 \mathrm{~b}$ & 12,1 \\
\hline Seção tronco $/ \mathbf{N}^{0}$ Frutos $\left(\mathrm{cm}^{2}\right.$.fruto $\left.{ }^{-1}\right)$ & $4,78 \mathrm{a}$ & $3,71 \mathrm{~b}$ & $4,59 \mathrm{a}$ & 18,54 & $3,99 \mathrm{~b}$ & $2,94 \mathrm{~b}$ & $6,15 \mathrm{a}$ & 30,1 \\
\hline Produtividade Estimada (ton.ha ${ }^{-1}$ ) & $15,06 \mathrm{~ns}$ & 16,74 & 15,05 & 17,9 & $14,12 \mathrm{~b}$ & $18,21 \mathrm{a}$ & $14,5 \mathrm{~b}$ & 27,06 \\
\hline Eficiência produtiva $\left(\mathrm{kg} . \mathrm{m}^{-3}\right)$ & $0,92 \mathrm{~ns}$ & 1,01 & 1,09 & 27,01 & $0,93 \mathrm{ab}$ & $1,22 \mathrm{a}$ & $0,87 \mathrm{~b}$ & 48,32 \\
\hline
\end{tabular}

*Médias seguidas pela mesma letra na linha não diferem entre si, pelo teste de Tukey, em nível de 5\% de probabilidade de erro. ** ns = não significativo

\section{CONCLUSÕES}

1-A poda verde com intensidade de $2 / 3$ de retirada dos ramos do ano aumenta a produtividade da pereira cv Abate Fetel, independentemente do mês de realização.

2-O porta-enxerto 'EMC' proporciona melhor controle de vigor para a cv Abate Fetel, resultando em melhores índices de produtividade.

\section{REFERÊNCIAS}

ASÍN, L.; ALEGRE, S.; MONTSERRAT, R. Effect of paclobutrazol, prohexadione-Ca, deficit irrigation, summer pruning and root pruning on shoot growth, yield, and return blomm, in a 'Blanquilla' pear orchard. Scientia Horticulturae, Amsterdam, v.113, p. 142-148, 2007.

BAKSHI, P.; SINGH, D.R. Rootstocks. In: SHARMA, R. M.; PANDEY, S. N.; PANDEY, V. (Ed.). The pear: production, post-harvest management and protection. India: IBDC Publishers, 2010. p. 147-161.

CALVO, G.; CANDAN, A.P.; GOMILA, T. PostHarvest Performance of 'Abate Fetel' Pears Grown in Argentina in Relation to Harvest Time, Acta Horticulturae, The Hague, n. 909, p.725-730, 2011.
GIACOBBO, C. L.; FACHINELLO, J. C.; PICOLOTTO, L.; Compatibilidade entre o marmeleiro porta-enxerto cv EMC e cultivares de Pereira. Scientia Agraria, Curitiba, v.8, n.1, p. 33-37, 2007.

HARTMANN, N.T.; KESTER, D.E.; DAVIES JUNIOR, F.T. Plant propagation: principles and practices. $5^{\text {th }}$ ed. Englewood Cliffs: Regents/PrenticeHall, 1990. 647 p.

IBRAF. INSTITUTO BRASILEIRO DE FRUTAS. Frutas frescas: importação. 2010. Disponível em: <www.ibraf.org.br>. Acesso em: 28 mar. 2011.

JACKSON, J.E. Biology of apples and pears. Cambridge:Cambridge University Press, 2003. 488 p.

MILOSEVIC, T.; MILOSEVIC, N. Influence of cultivar and Rootstock on early growth and syllepsis in nursery tress of pear (Pyrus communis L., Rosaceae). Brazilian Archives Biology and Technology, Curitiba, v. 54, n. 3, p. 451-456, 2011.

PEREIRA, J. F. M.; HERTER, F. G. Tecnologias para o aumento da produtividade e regularidade de produção de pera na região Sul do Brasil. In: REUNIÃO TÉCNICA DA CULTURA DA PEREIRA: BUSCA PELA IDENTIDADE NACIONAL, 3., 2010, Lages. Anais... p. 39-45. 
PREDIERI, S.; GATTI, E. Effects of cold storage and shelf-life on sensory quality and consumer acceptance of 'Abate Fetel' pears. Postharvest Biology and Technology, Amsterdam, v.51, p. 342-348, 2009.

PREDIERI, S., GATTI, E., RAPPARINI, F., CAVICCHI, L., COLOMBO, R.. Sensory evaluation from a consumer perspective and its application to 'Abate Fetel' pear fruit quality. Acta Horticulturae, The Hague, n. 671, p. 349-353, 2005.

PIO, R.; CHAGAS, E. A.; BARBOSA, W.;SIGNORINI, G.; ENTELMANN, F.A.; FIORAVANÇO, J. C.; FACHINELLO, J. C.; BIANCHI, V.J. Desenvolvimento de 31 cultivares de marmeleiro enxertadas no porta-enxerto 'Japonês'. Revista Brasileira de Fruticultura, Jaboticabal, v. 30, n.2, p. 466-470. 2008.
RUFATO, L.; KRETZSCHMAR, A. A.; BOGO, A.; MACHADO, B. D.; MARCON FILHO, J. L.; LUZ,A. R.; MARCHI, T. Vegetative Aspects of European Pear Scions Cultivars in Combination with Quince Rootstocks in Urupema Santa Catarina State, Brazil. Acta Horticulturae, The Hague, n. 909, p. 207-213, 2011.

SOARES, J.; SILVA, A.; MARQUES, H. O livro de pera rocha: intensificação cultural e regulação da produção. 2.ed. Cadaval: Associação Nacional de Produtores de Pera Rocha, 2003. v.2, 192 p.

STERN, R.A.; DORON, I. Performance of 'Coscia' pear (Pyrus communis) on nine rootstocks in the north of Israel. Scientia Horticulturae, Amsterdam, v.119, p. 252-256, 2009.

TREVISAN, R.; CHAVARRIA, G.; HERTER, F. G.; GONÇALVES, E. D.; RODRIGUES, A. C.; VERÍSSIMO, V.; PEREIRA, I. S. Raleio de gemas florais para a redução do abortamento em pereira (Pyrus pyrifolia) na região de Pelotas-RS. Revista Brasileira de Fruticultura, Jaboticabal, v. 27, n.3, p. 504-506, 2005. 